es denkbar, daß aufgrund lipophiler oder hydrophiler Eigenschaften eine gewisse Blut-Liquor Schranke für freie und konjugierte Steroide besteht, wobei Steroidsulfatide als lipophile Konjugate gegenüber den hydrophilen Steroid-glucuronosiden eine bevorzugte Stellung einnehmen könnten.

Was die Identität der untersuchten $\mathrm{C}_{18^{-}}, \mathrm{C}_{19^{-}}$und $\mathrm{C}_{21^{-}}$ steroide angeht, so gestatten die angewandten chromatographischen Trennverfahren eine einwandfreie Isolierung der Einzelverbindungen mit Ausnahme von Pregnenolon und Androsteron - letztere Steroide wurden in ali- quoten 'Teilen des gemeinsamen Eluats mittels geeigneter Farbreaktionen bestimmt - die im Verein mit den mehr oder weniger spezifischen Farbreaktionen einen ausreichenden qualitativen und quantitativen Nachweis gewährleisten sollten.

Hinsichtlich der physiologischen Bedeutung von freien und konjugierten Steroiden im menschlichen Liquor erhebt sich schließlich die Frage, ob diesen im Regulationsmechanismus für Steroide innerhalb des HypophysenNebennierenrinden(Gonaden)-Systems eine bestimmte Rolle zukommt.

\title{
Literatur
}

1. Sandberg, A. A., K. B. Eik-Nes, D. H. Nelson und F. H. Tyler, J. Laborat. Clin. Med., S. Louis 43, 874 (1954). - 2. Abelson, D., D. N. Baron und J. G. Toakley, J. Endoct. 12, 87 (1955). - 3. Ianniruberto, A., G. Marandola und S. Parisi, Minerva pediatr., Torino 13, 746 (1961). - 4. Oertel, G. W. und E. KAISER, Biochem. Z. 336, 10 (1952). - 5. Burstein, S. und S. Lieberman, J. biol. Chemistry 233, 331 (1959). - 6. Engel. L. L. und I. T. Nathanson, J. biol. Chemistry 185, 255 (1950). 7. Lisboa, B. P. und E. Drczfalusy, Acta endocr. K'hvn 40,60 (1962); 43, 545 (1963). - 8. Struck, H., Mikrochim. Acta, 634 (1961). - 9. LrsBoA, B. P., Acta endocr. K'hvn 43, 47 (1963). -
10. Savard, K., J. biol. Chemistry 202, 457 (1953). - 11. Itrrich. G., Hoppe-Seyler's Z. physiol. Chem. 312, 1 (1958). - 12. ZIMMERMANN, W., Hoppe-Seyler's Z. physiol. Chem. 233, 257 (1935). - 13. Ceresa, F. und C. A. Cravetro, Acta endocr. K'hvn. 29, 321 (1958). - 14. Oertel, G. W. und K. B. Eik-Nes, Analytic Chem. 31, 98 (1959). - 15. Carstensen, H., G. W. Oertel, und K. B. Erk-Nes, J. biol. Chemistry 234, 2570 (1959). - 16. BraunsBerg, H. und V. H. T. James, J. endocr. 21, 327 (1960). 17. Kornel, L., Metabolism 11, 1019 (1962). - 18. Schwarz, K., Dtsch. Zschr. Nervenhk. 177, 464 (1958).

Dozent Dr. G. W. Oertel Universität des Saarlandes, Inst. für Hygiene und Mikrobiologie 665 Homburg (Saar)

\section{Zum Nachweis von 5-Carbamyl-5 H-dibenzo[b, f] azepin im Liquor cerebrospinalis mittels Dünnschichtchromatographie}

\author{
Von F. Scheiffarth, F. Weist und L. Zicha \\ Aus der Medizinischen Klinik der Universität Erlangen-Nïrnberg (Direktor: Prof. Dr. med. N. Henning)
}

(Eingegangen am 14. August 1965)

\begin{abstract}
Es wurde ein empfindliches, dünnschichtchromatographischessNachweisverfahren für 5-Carbamyl-5H-dibenzo [b, $\mathrm{f}]$ azepin ausgearbeitet, mit dessen Hilfe es gelang, diese Substanz im Liquor und anderen Körperflüssigkeiten nachzuweisen. Zwei im Liquor auftretende Metaboliten des „Tegretal ®“ konnten außerdem charakterisiert werden. Auf Grund der vorliegenden Untersuchungsergebnisse zeigt sich eine erhebliche Affinität des „Tegretal“ zum Hirngewebe, was auch durch den Nachweis dieser Substanz im Leichengehirn unter Beweis gestellt werden konnte.

A sensitive, thin layer chromatographic method for the detection of 5-carbamyl-5H-dibenzo [b, $f$ ] azepin was devised. It was used to demonstrate this substance in cerebrospinal fluid and other body fluids. Furthermore, two metabolites of "Tegretal", which appear in the CSP, were characterised. The experimental results show that "Tegretal" has a high affinity for brain tissue, which was confirmed by the demonstration of this substance in post mortem brain.
\end{abstract}

Eine Reihe von günstigen therapeutischen Effekten bei der Behandlung der Epilepsie, der Trigeminusneuralgie und des Diabetes insipidus im Ablauf einer Röntgenbestrahlung von Hypophysentumoren und schließlich auch bei der Hirsutismustherapie (1), ließ darauf schlieBen, daß 5-Carbamyl-5H-dibenzo $[b, f]$ azepin $\left.{ }^{1}\right)$ die Blut-Liquorschranke zu passieren vermag. Erfahrungsgemäß sind jedoch die Konzentrationen von Pharmaka im Liquor oft noch niedriger als im Blut (2), so daß die Entwicklung sehr empfindlicher Nachweismethoden für

1) Tegretal®; Herstellerfirma: J. R. Geigy A. G., Basel; PharmaHerstellung und Vertrieb für Deutschland: Fa. Dr. Karl Thomae $\mathrm{GmbH}$, Biberach an der Riss. die Bearbeitung der vorgesehenen Fragestellungen notwendig wurde. Die Dünnschichtchromatographie bringt einerseits eine untere Bestimmungsgrenze von 0,1 bis $0,001 \mu \mathrm{g}$, andererseits hat sie sich auch für den quantitativen Nachweis von zahlreichen physiologischen und pharmakologischen Substanzen sowie zur Isolierung von Metaboliten bewährt (3-6).

\section{Methodik}

Für die dünnschichtchromatographische Auftrennung kamen die Originalmethode nach STAHL (3) sowie quantitative und präparative Modifikationen zur Anwendung. Als Sorptionsschicht wurde Kieselgel-G, 
Fa. Merck, Darmstadt mit einem Zusatz von Leuchtpigment ZS Super, Riedel de Haen, Seelze verwendet.

Tegretal besitzt nach Besprühen mit 70-proz. Perchlorsäure und anschließendem Erhitzen auf $120^{\circ}$ für 20 Minuten eine derartig große spezifische Fluoreszenz, daß selbst Mengen von $2 \times 10^{-8} \mathrm{~g}$ im filtrierten UV-Licht bei $360 \mathrm{~m} \mu$ noch nachweisbar sind. Diese Tatsache gestattet es, biologische Flüssigkeiten, wie z. B. Liquor, direkt ohne irgendwelche Anreicherungs- oder Extraktionsverfahren mittels der Dünnschichtchromatographie auf ihren Gehalt an Tegretal hin zu untersuchen. Jedoch ist hierbei eine Konzentration von $2 \times 10^{-7} \mathrm{~g} / \mathrm{ml}$ Voraussetzung und stellt die untere Nachweisgrenze von Tegretal in wenig Begleitstoffe enthaltenden biologischen Flüssigkeiten dar. Ist die Konzentration der bei der chromatographischen Trennung störenden Begleitstoffe zu hoch, wie z. B. in der Galle, oder liegt jene von Tegretal unter dem oben angegebenen Wert, so ist eine Extraktion erforderlich. Von den mehr als 10 getesteten Extraktionsmitteln erscheinen hierbei 1,2-Dichloräthan, andere niedrige chlorsubstituierte Kohlenwasserstoffe und Äthylacetat gleichwertig. Für 1,2-Dichloräthan sprechen verfahrenstechnische Vorteile, $z$. B. sein gegenüber Wasser niedrigeres spezifisches Gewicht und gegenüber Äthyalcetat niedrigerer Siedepunkt. Die nach dreimaligem Ausschütteln erhaltenen Extrakte wurden bei $40^{\circ}$ unter schwachem Unterdruck zur Trokkene eingeengt, in 1,2-Dichloräthan erneut aufgenommen und in der jeweils gewünschten Menge auf verschiedene Dünnschichtplatten aufgetragen und entwickelt. - Von den über 70 getesteten Steigmitteln hat sich schließlich Tetrachlorkohlenstoff : Methanol im Verhältnis 75:25 am günstigsten erwiesen.

Für die quantitative Auswertung wurden Bandchromatogramme angefertigt, wobei zunächst Reinsubstanz und Zusatzstoffe am Rande der Dünnschichtplatten der Lokalisation des Tegretal und der Metaboliten dienten. Die so lokalisierten Substanzen wurden mit 70-proz. Perchlorsäure aus dem abgekratzten Kieselgel eluiert, auf $120^{\circ}$ für die Dauer von 20 Minuten erhitzt und anschließend mit dem ZEIssschen Fluoreszenzspektrophotometer bestimmt. Das Anregungsmaximum lag bei $358 \mathrm{~m} \mu$, das Emissionsmaximum bei $498 \mathrm{~m} \mu$, die Spaltbreite betrug für den Eingangsspalt 0,5, für den Emissionsspalt $0,4 \mathrm{~cm}$, die Verstärkung 2/1/7/4. Die Messung erfolgte gegen einen Reagenzienleerwert. Es hat sich jedoch gezeigt, daß es für die oben beschriebene Bestimmungsmethode und die hiermit gewonnene Eichkurve ohne Einfluß bleibt, ob zur besseren Lokalisation von Tegretal und seinen Metaboliten bereits vor dem Abkratzen und der Elution mit Perchlorsäure angefärbt wurde oder nicht. Die Eichkurve für beide Veifahren ist in Abbildung 1 dargestellt.

\section{Ergebnisse}

Mit Hilfe dieses Verfahrens wurden über 30 Liquores nach oraler Applikation von Tegretal in Dosen von 600 bis $1200 \mathrm{mg} / \mathrm{Tag}$ auf ihren Gehalt an dieser Substanz

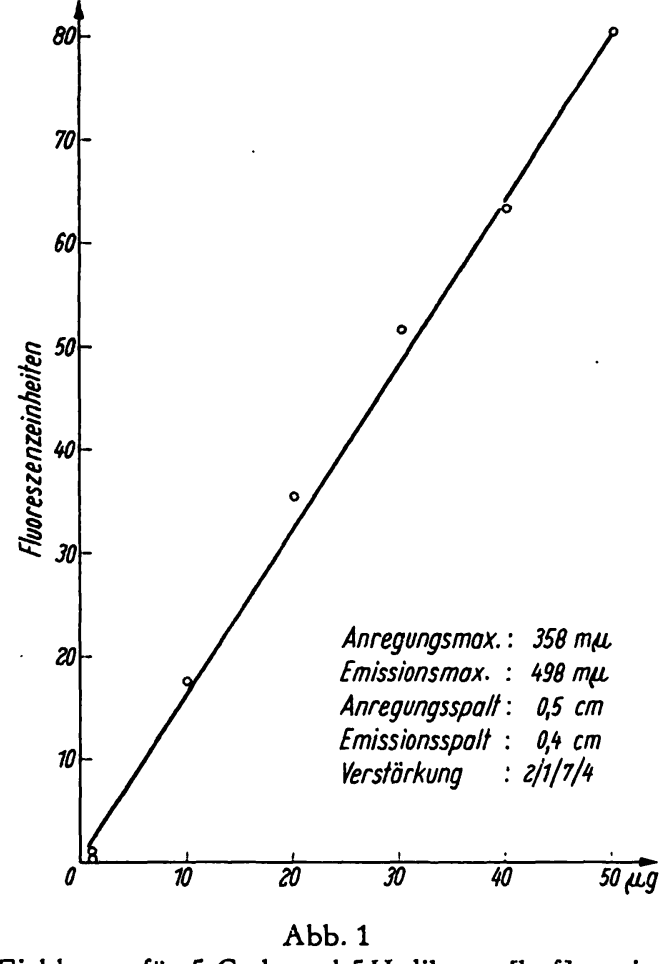

Eichkurve für 5-Carbamyl-5H-dibenzo[b, f]azepin

untersucht. Die Liquorpunktionen erfolgten jeweils 1-144 Stunden nach Verabreichung.

Nach oraler Applikation von $1200 \mathrm{mg}$ Tegretal fand man sowohl 5-Carbamyl-5H-dibenzo[b, f]azepin als auch zwei Metaboliten im Liquor. $R_{\mathrm{F}}$-Werte und Anfärbbarkeit von Tegretal und seiner Metaboliten sind in Tabelle 1 zusammengefaßt. Bei der quantitativen Bestimmung der Metaboliten wurden zunächst die Werte auf 5-Carbamyl-5H-dibenzo[b, $f]$ azepin bezogen, da eine Identifizierung dieser Substanzen bisher nicht gelang.

Tab. 1

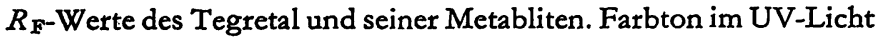
bei $360 \mathrm{~m} \mu$ und nach $\mathrm{HClO}_{4}$-Färbung: grünlich

\begin{tabular}{ll}
\hline \multicolumn{1}{c}{ Substanz } & $R_{\text {F-Wert }}$ \\
\hline 5-Carbamyl-5H- & \\
dibenzo(b, f)azepin & 0,60 \\
Metabolit II & 0,50 \\
Metabolit I & 0,37 \\
\hline
\end{tabular}

Vergleicht man die Intensitäten des Tegretal und seiner Metaboliten im Einzelfalle, so findet sich eine unterschiedliche Menge der einzelnen Komponenten, wobei sich zunächst keine Beziehungen zwischen der Diagnose und dem Auftreten der beiden Metaboliten nachweisen lassen. Der Verbleib des Tegretal und seiner Stoffwechselprodukte im Liquor nach Absetzen der Therapie weist insofern auf eine Bindung im cerebrospinalen System hin, als 1 . die Konzentration im Liquor zum Teil über der des Blutes liegt, 2. noch 6 Tage nach Behandlungsende nachweisbare Mengen im Liquor festzustellen sind und 3. im Hirngewebe des Sektionspräparates eines Patienten mit Hilfe qualitativer dünnschichtchromatographischer Methoden Tegretal gefunden werden kann. 


\section{Diskussion}

Die vorliegenden Untersuchungsergebnisse haben gezeigt, daß die Blut-Liquorschranke nicht nur für 5-Carbamyl-5H-dibenzo[b, f]azepin, sondern auch für die zwei beschriebenen Metaboliten durchgängig sein muß. Auf Grund von Beobachtungen, wie sie sich bei der qualitativen dünnschichtchromatographischen Untersuchung anderer Körperflüssigkeiten, wie Serum, Harn und Galle ergaben, muß angenommen werden, $\mathrm{da} \beta$ die beiden Metaboliten hier ebenfalls vorkommen. Hierüber, sowie über die Pharmakodynamik des Tegretal, d. h. über seine Konzentration in Blut, Galle, Liquor und Harn in Abhängigkeit von der Art und dem Zeitpunkt der Applikation, wird ausführlich an anderer Stelle berichtet (1).
Die relativ einfache Methodik und insbesondere ihre große Empfindlichkeit erlauben außerdem, die Verteilung des Tegretal in Organen, z. B. auch im Hirn, ohne Anwendung der Isotopentechnik zu bestimmen. Inwieweit allerdings die zentralen Effekte des Tegretal allein auf die Substanz oder auf einen der entstandenen Metaboliten zurückzuführen sind, muß vorerst noch offen bleiben. Die gute Liquorgängigkeit, sowie die Affinität zum Hirngewebe und der günstige therapeutische Effekt, insbesondere bei Erkrankungen mit Quellungsprozessen bzw. lokalen Ödemreaktionen im Gehirn, legen die Vermutung nahe, daß Tegretal einen Permeabilitätseffekt in diesem Bereich entfaltet. Allerdings besitzt diese Annahme nur den Wert einer Arbeitshypothese. Ihre Gültigkeit blẹibt zu beweisen.

\title{
Literatur
}

1. Braunhofer, J., F. Weist, L. Zicha und E. Schmid, Ergebnisse klinischer und klinisch-chemischer sowie elektrophoretischer Untersuchungen über ein neues Dibenzazepinderivat. 8th Intern. Congress of Neurology, Wien, September 5.-10., 1965. - 2. Zicha, L., F. Freytag, F. Werst, Atzneimittel-Forsch., Aulendorf 15, 777 (1965). - 3. STAHL, E., Dünnschichtchromatographie, ein
Laboratoriumshandbuch. Springer-Verlag, Berlin-Göttingen-Heidelberg (1962). - 4. TAUTZ, N. A., G. VotTmER und E. Schmid, Klin. Wschr. 43, 233 (1965). - 5. WALdr, D., Klin. Wschr. 40, 827 (1962). - 6. ZiCHA, L., F. WEIST, F. SChEIFFARTH und E. SCHMID, Arzneimittel-Forsch., Aulendorf 14, 699 (1964).

Professor Dr. med. F. Scheiffarth Med. Klinik der Universität 852 Erlangen, Krankenhausstr. 12

\section{Continuous "in vivo" registration of bromsulfalein disappearance curves}

\author{
By J. Versieck, A. Elewaut and F. Barbier \\ From the University of Ghent, Policlinic for Internal Medicine (Director: Prof. Dr. L. Re mouchamps) \\ Akademisch Ziekenbuis, Gent, Belgium
}

(Eingegangen am 30. Juli 1965)

\begin{abstract}
The reliability of the automated continuous "in vivo" registration of BSP with AutoAnalyzer is tested. Impairment of dialysis occurs when $0,80 \mathrm{~m} / / \mathrm{min}$ blood is aspirated as recommended by other authors. When a BSP solution with constant concentration in blood, plasma or serum is registered making use of this manifold, a progressive decrease of extinction is obtained and therefore the calculated concentration becomes falsely low. It is not impossible that the same phenomenon can be found with technics for other continuous registrations when relatively great amounts of blood are aspirated. The performed experiments prove the necessity of using a tube with small delivery (e. g. 0,32 or $0,16 \mathrm{~m} / / \mathrm{min}$ ) for aspiration of sample.

Die Zuverlässigkeit des Verfahrens dex automatischen Dauerregistrierung von BSP ,in vivo“ mit dem Autoanalyzer wird getestet. Eine Verschlechterung der Dialyse entsteht, wenn 0,8 m//Min. Blut aspiriert wird, wie andere Autoren es empfehlen. Wenn eine BSP-Lösung mit konstanter Konzentration in Blut, Plasma oder Serum registriert wird mit diesem Fließ-Schema, wird eine progressive Abnahme der Extinktion erhalten, und deshalb wird die Konzentration fälschlich zu niedrig bewertet. Es ist nicht unmöglich, daß das gleiche Phänomen auch bei Techniken für andere fortlaufende Registrierungen gefunden werden kann, wenn verhältnismäßig große Blutmengen aspiriert werden. Die ausgeführten Versuche beweisen die Notwendigkeit, Leitungen zu verwenden, die kleine Mengen (z. B. 0,32 oder 0,16 m//Min.) für die Aspiration der Einzelprobe liefern.
\end{abstract}

The automated continuous "in vivo" registration of bromsulfalein ("BSP") seems a promising method for investigation of hepatic function in health and disease. The object of the present study was to analyse some factors which can interfere with the reliability of the method.

\section{Methods and results}

We made use of the AutoAnalyzer manufactured by Technicon Instruments Corporation. The flow diagram is represented in figure 1 . A flow cuvette of $15 \mathrm{~mm}$ light path being available, a range expander and a double set of dialysis plates (1) seemed unnecessary when $0,80 \mathrm{~m} /$ min. sample is aspirated. The former was only used when the sample flow was reduced to 0,32 or $0,16 \mathrm{~m} /$ $\min$.

Two different manifolds (one being the unchanged manifold according to GALLI et al. [1]) - having in common an identical sample-tube with delivery $0,80 \mathrm{~m} /$ min. but differing by the flow of alkaline reagent ${ }^{1}$ ) and

1) Alkaline reagent : sodium hydroxide $2,0 \mathrm{~g}$; sodium chloride $7,0 \mathrm{~g}$; sodium p-toluenesulfonate $6,4 \mathrm{~g}$; twice-distilled water $1000 \mathrm{~m}$ l. 